\title{
Simultaneous Thrombosis in a Normal Left Ventricle and Normal Carotid Artery in a Patient With a Stroke Secondary to Iron Deficiency Anemia
}

\author{
Tomoki Nakamizo $^{\mathrm{a}, \mathrm{d}}$, Kiyoko Ishikawa ${ }^{\mathrm{b}}$, Kazumitsu Amaric $^{\mathrm{c}}$
}

\begin{abstract}
Iron deficiency anemia (IDA) is implicated as a cause of stroke, particularly in young patients without cardiovascular disease. In such patients, thrombi sometimes form in carotid arteries or the aorta. We report here a patient with a stroke secondary to IDA with thrombi in the normal left ventricle and normal carotid artery. The patient was a 45-year-old woman with severe IDA who developed cerebral infarction in the right middle cerebral artery. She had no other thrombophilia or cardiovascular diseases. Echocardiography showed a left ventricular thrombus without cardiac disease, and the carotid ultrasound showed a mobile thrombus attached to the right internal carotid artery without atherosclerosis. Antithrombotic therapy with iron supplementation removed both thrombi within 2 weeks. This is the first case of IDA with a ventricular thrombus in a normal heart. This case identifies a new site for thrombosis in IDA and shows that patients with IDA may present with simultaneous thrombosis at separate sites. IDA must therefore be recognized as a cause of stroke or systemic embolism, particularly in patients without detectable cardiovascular disease. In such patients, it is important to conduct careful investigations to detect thrombosis.
\end{abstract}

Keywords: Carotid artery thrombosis; Echocardiography; Embolism; Iron deficiency anemia; Left ventricular thrombosis; Menorrhagia; Stroke; Cardiac thrombosis; Cerebral infarction

Manuscript accepted for publication May 5, 2014

${ }^{\text {a } D e p a r t m e n t ~ o f ~ N e u r o l o g y, ~ H i r a t s u k a ~ C i t y ~ H o s p i t a l, ~ 1-19-1 ~}$

Minamihara, Hiratsuka, Kanagawa, 254-0065, Japan

${ }^{\mathrm{b}}$ Department of Clinical Laboratory, Kitakashiwa Rehabilitation

General Hospital, 265 Kashiwashita, Kashiwa, Chiba, 277-0004, Japan

${ }^{c}$ Department of Neuro-endovascular Therapy, Yokohama Stroke and

Brain Center, 1-2-1 Takigashira, Isogo-ku, Yokohama, Kanagawa,

235-0012, Japan

${ }^{\mathrm{d}}$ Corresponding Author: Tomoki Nakamizo, Department of Neurology,

Hiratsuka City Hospital, 1-19-1 Minamihara, Hiratsuka, Kanagawa,

254-0065, Japan. Email: nakamizo@kuhp.kyoto-u.ac.jp

doi: http://dx.doi.org/10.14740/jmc1786w

\section{Introduction}

Iron deficiency anemia (IDA) is a common disease implicated as an uncommon cause of stroke and is epidemiologically associated with increased risk of stroke $[1,2]$. Moreover, several case reports suggest a causative role of IDA in young and otherwise healthy patients with stroke or with systemic embolism where thrombi are sometimes detected on an apparently intact arterial wall such as the carotid artery [3-9] or aorta $[10,11]$. Here we describe a patient with thrombi in an otherwise normal carotid artery and left ventricle, which is a previously unreported site for thrombosis in patients with IDA. Our case exemplifies a new site for thrombosis in IDA and indicates that patients with IDA may present with simultaneous thrombosis at separate sites.

\section{Case Report}

A 45-year-old woman presented with left hemiparesis. Three years before admission she was diagnosed with IDA, which was attributed to menorrhagia; however, she did not take medication and was otherwise healthy. She experienced headache and left hemiparesis upon awakening and was admitted to the hospital. Her blood pressure was $122 / 80 \mathrm{~mm}$ $\mathrm{Hg}$, pulse rate regular at $90 / \mathrm{min}$ and anemic conjunctiva. She was alert but slightly agitated. Neurological examination revealed left hemiparesis and left hemispatial neglect, and magnetic resonance (MR) imaging revealed right frontoparietal infarction. MR angiography revealed occlusion of the distal branches of the right middle cerebral artery, and MR venography revealed normal dural sinuses. The electrocardiogram was normal. Hemoglobin was $6.0 \mathrm{~g} / \mathrm{dL}$, mean corpuscular volume $65.3 \mathrm{fL}$, platelets $573,000 / \mu \mathrm{L}$, serum iron $19 \mu \mathrm{g} / \mathrm{dL}$, total iron-binding capacity $497 \mu \mathrm{g} / \mathrm{dL}$, transferrin saturation $3.8 \%$ and ferritin $1.6 \mathrm{ng} / \mathrm{mL}$. Protein S, protein C, antithrombin III and antiphospholipid antibody values were normal, indicating the absence of thrombophilia.

The next day, transthoracic echocardiography revealed a mobile thrombus in the left ventricle apparently attached to the chordae tendineae of the mitral valve (Fig. 1). Chamber 


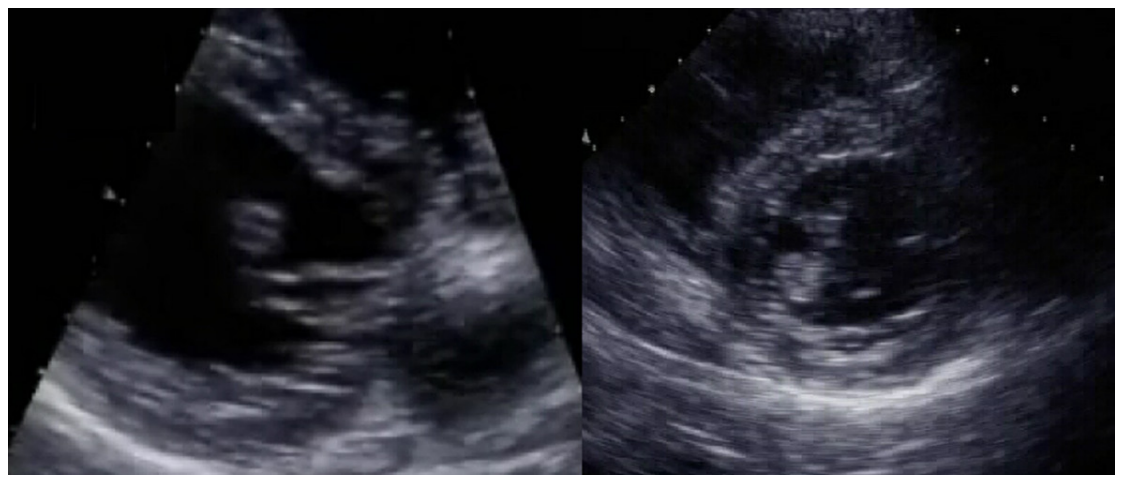

Figure 1. Transthoracic echocardiogram on day 2 reveals a left ventricular thrombus. The thrombus is slightly hypoechoic compared with the myocardium and shows an irregular flapping movement. It is apparently attached to the chordae tendineae of the mitral valve. Left: parasternal long axis view. Right: parasternal short axis view, mid-ventricle level slightly deviated to the base.

size, wall motion and cardiac valves were normal. Carotid ultrasound revealed a mobile thrombus attached to the wall of the right internal carotid artery (Fig. 2). There was no detectable atherosclerotic lesion with a maximal intima-media thickness of $1.0 \mathrm{~mm}$. She was treated with aspirin, clopidogrel and argatroban (a direct thrombin inhibitor) as well as iron supplementation. Her symptoms resolved after 2 weeks, and no thromboembolism occurred after admission.

On day 4 of hospitalization, ultrasound revealed that the carotid thrombus was reduced in size by $50 \%$, and on day 8 , carotid ultrasound did not detect a thrombus. On day 11 , transesophageal echocardiography did not detect a cardiac thrombus; no septal defect or patent foramen ovale was present, and the thoracic aorta was normal. On day 14, transthoracic echocardiography revealed no left ventricular thrombus.

\section{Discussion}

IDA is implicated as a cause of stroke or systemic thromboembolism, and epidemiological studies [1,2] revealed that IDA is more prevalent in patients with stroke compared with controls. Moreover, when IDA is associated with stroke or a systemic embolism, large thrombi are sometimes present in an apparently intact carotid artery [3-9] as in our present case or less commonly in the aorta $[10,11]$. These patients are young women 20 - 50 years of age with severe IDA ( $\mathrm{Hb} 5.5$ - $10 \mathrm{~g} / \mathrm{dL}$ ), which is often caused by menorrhagia. More important, they have no known cardiovascular disease. These common features, taken together with the characteristics of our present patient, suggest that IDA plays a causative role in certain young patients with stroke rather than as a risk factor.

The present case reveals that the left ventricle represents a new site for thrombosis in patients with IDA. In the absence of detectable cardiac disease, left ventricular thrombosis is rare and described only anecdotally. Many of these patients have prothrombotic disorders such as protein $\mathrm{C}$ deficiency [12], protein S deficiency [13], essential thrombocythemia [14] and antiphospholipid antibody syndrome [15]; however, there are no reports of patients with left ventricular thrombus associated with IDA. Because our patient had no other detectable prothrombotic disorders, we concluded that IDA was the sole cause of left ventricular thrombosis. Moreover, since left ventricular thrombosis is rare in the absence of cardiac disease, such a thrombus may be overlooked, particularly when it is small or less mobile. Conversely, when the thrombus is large and mobile, it may be indistinguishable from cardiac myxoma [14-17], and this diagnostic uncertainty may lead to surgery. In our present patient, the thrombus was distinguished from myxoma by its relatively small size and its resolution after treatment. Therefore, it is important to recognize IDA as a cause of left ventricular thrombosis to avoid unnecessary surgery as well as to identify the embolic source.

The characteristics of our present patient, taken together

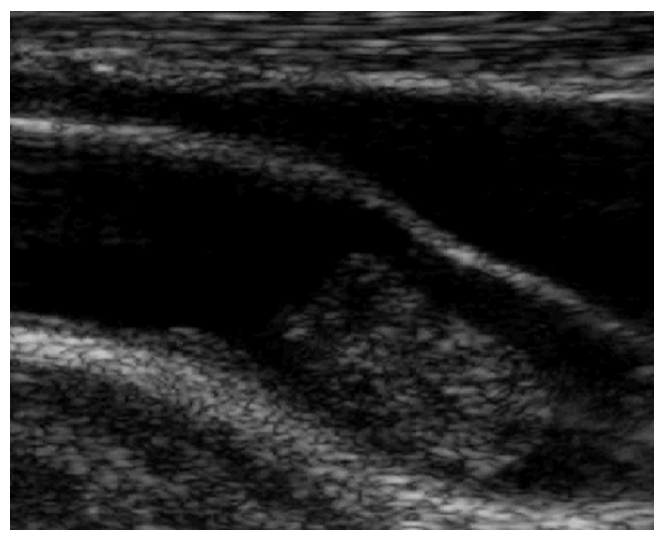

Figure 2. Carotid ultrasound on day 2 reveals a large mobile thrombus attached to the wall of the right internal carotid artery. There is no atherosclerotic change. 
with those of others [3-11], suggest that in patients with IDA, a thrombus may be formed proximally from the left ventricle to the carotid arteries. In view of Virchow's triad for thrombosis, it is surprising that a massive thrombus was formed in apparently intact large arteries such as the aorta or carotid where the blood flows without stagnation. A fortiori, thrombosis in the normal left ventricle appears surprising. How a thrombus is formed at such sites is unclear, although reactive thrombocytosis may be involved [3, 18-20]. Further, Akins et al [3] speculate that thrombosis may be caused by endothelial injury, which in turn is caused by turbulent blood flow. They state further that the Reynolds number may exceed the threshold for turbulence because IDA decreases viscosity and increases blood velocity. The Reynolds number, which equals $\mathrm{rVd} / \mathrm{n}$, where $\mathrm{r}$ is the radius, $\mathrm{V}$ is the velocity, $\mathrm{d}$ is the density and $\mathrm{n}$ is the viscosity, explains why IDA induces turbulence by decreasing viscosity and increasing velocity. Moreover, this may explain why thrombi are often formed in large arteries because the Reynolds number is proportional to the radius of the artery.

We detected thrombi in our patient's proximal internal carotid artery and left ventricle. When a cardiac embolus lodges in carotid arteries, it usually leads to the occlusion of the distal intracranial internal carotid arteries [21]. On the contrary, the thrombus in our patient was loosely attached to the proximal internal carotid wall without occlusion, which suggests in situ thrombosis rather than embolism. Therefore, we believe that the thrombi were formed independently in the carotid artery and left ventricle. There is a report of thrombosis at separate sites in only one patient with IDA [22] who presented with simultaneous arterial and venous cerebral infarctions, although other prothrombotic coagulation disorders were present. The present patient indicates that a thrombus can be formed simultaneously at separate sites and serves to emphasize the importance of careful investigation to detect the embolic source.

In the present and previous cases, treatment included anticoagulation, antiplatelet therapy and iron supplementation. Our patient's thrombi were undetectable 8 - 11 days after treatment was initiated. Similar early resolution, as early as 7 days, was observed in other patients $[3,11]$. Although no randomized trials were conducted, these treatments are reasonable and appear effective.

In some patients with stroke $[18,19,23]$ or with systemic embolism [20] associated with IDA, no embolic source was found, although they share features similar to ours, specifically young women with menorrhagia, very low hemoglobin level and without cardiovascular disease. In these cases, undetected thrombi may have formed in the left ventricle, aorta, or carotid arteries. Early resolution or unexpected sites such as the left ventricle or aorta may explain why thrombi were not detectable.

In addition to arterial thromboembolic disease, IDA is associated with cerebral venous thrombosis $[24,25]$. There- fore, IDA may contribute to thrombosis by different mechanisms and may be involved in various forms of thrombotic disease. Therefore, IDA should be recognized as an important cause of stroke.

In conclusion, IDA must be recognized as a cause of stroke or systemic embolism, particularly in young patients without detectable cardiovascular disease. In such patients, thrombi can form simultaneously at separate sites, including the normal left ventricle. Therefore, IDA patients should be examined carefully for thrombosis.

\section{Grant Support}

None.

\section{Conflict of Interest}

All the authors have no conflict of interest related to this study.

\section{References}

1. Dubyk MD, Card RT, Whiting SJ, Boyle CA, Zlotkin $\mathrm{SH}$, Paterson PG. Iron deficiency anemia prevalence at first stroke or transient ischemic attack. Can J Neurol Sci. 2012;39(2):189-195.

2. Chang YL, Hung SH, Ling W, Lin HC, Li HC, Chung $\mathrm{SD}$. Association between ischemic stroke and iron-deficiency anemia: a population-based study. PLoS One. 2013;8(12):e82952.

3. Akins PT, Glenn S, Nemeth PM, Derdeyn CP. Carotid artery thrombus associated with severe iron-deficiency anemia and thrombocytosis. Stroke. 1996;27(5):10021005.

4. Batur Caglayan HZ, Nazliel B, Irkec C, Dumlu A, Filiz A, Panpalli Ates M. Iron-Deficiency Anemia Leading to Transient Ischemic Attacks due to Intraluminal Carotid Artery Thrombus. Case Rep Neurol Med. 2013;2013:813415.

5. Caplan L, Stein R, Patel D, Amico L, Cashman N, Gewertz B. Intraluminal clot of the carotid artery detected radiographically. Neurology. 1984;34(9):1175-1181.

6. Yarnell P, Earnest M, Kelly G, Sanders B. Disappearing carotid defects. Stroke. 1978;9(3):258-262.

7. Keung YK, Owen J. Iron deficiency and thrombosis: literature review. Clin Appl Thromb Hemost. 2004;10(4):387-391.

8. Bouly S, Le Bayon A, Blard JM, Touze E, Leys D, Mas JL, Neau JP, et al. [Spontaneous thrombosis of lesionfree carotid arteries: a retrospective analysis of eight patients]. Rev Neurol (Paris). 2005;161(1):61-66. 
9. Switzer JA, Nichols FT. Teaching NeuroImages: disappearing intraluminal thrombus of the carotid artery in reactive thrombocytosis. Neurology. 2011;76(16):e7879.

10. Bukharovich IF, Wever-Pinzon O, Shah A, Todd G, Chaudhry FA, Sherrid MV. Arterial embolism caused by large mobile aortic thrombus in the absence of atherosclerosis, associated with iron deficiency anemia. Echocardiography. 2012;29(3):369-372.

11. Yakushiji Y, Terasaki Y, Otsubo R, Yasaka M, Oe H, Yamada N, Nishigami K, et al. Brain embolism caused by a mobile aortic thrombus with iron deficiency anemia. Cerebrovasc Dis. 2005;20(6):475-478.

12. Sivasankaran S, Harikrishnan S, Tharakan JM. Left ventricular thrombi in the presence of normal left ventricular function. Indian Heart J. 2002;54(2):196-198.

13. Kawamoto J, Ishibashi K, Shibukawa T, Izutani H. Left ventricular thrombus with a normal heart. Gen Thorac Cardiovasc Surg. 2007;55(8):322-324.

14. Allende NG, Sokn F, Borracci R, Milani A, Kusselevski A, Camilletti J, Tronge J, et al. Giant pedunculated thrombus with normal left ventricular systolic function mimicking myxoma. Echocardiography. 2011;28(2):E31-33.

15. Cianciulli TF, Saccheri MC, Lax JA, Neme RO, Sevillano JF, Maiori ME, Lound SF, et al. Left ventricular thrombus mimicking primary cardiac tumor in a patient with primary antiphospholipid syndrome and recurrent systemic embolism. Cardiol J. 2009;16(6):560-563.

16. Jeganathan R, Ralph-Edwards A. Left apical pedunculated thrombus with normal ventricular function mimicking an intracardiac tumor. J Card Surg. 2011;26(6):614-
616.

17. Yadava OP, Yadav S, Juneja S, Chopra VK, Passey R, Ghadiok R. Left ventricular thrombus sans overt cardiac pathology. Ann Thorac Surg. 2003;76(2):623-625.

18. Saxena VK, Brands C, Crols R, Moens E, Marien P, de Deyn PP. Multiple cerebral infarctions in a young patient with secondary thrombocythemia due to iron deficiency anemia. Acta Neurol (Napoli). 1993;15(4):297-302.

19. Scoditti U, Colonna F, Ludovico L, Trabattoni G. Mild thrombocytosis secondary to iron-deficiency anemia and stroke. Riv Neurol. 1990;60(4):146-147.

20. Mall T, Schmitt HE, Widmer LK. Arterial occlusions in iron deficiency anemia with thrombocytosis. Vasa. 1985;14(2):188-190.

21. Helgason CM. Cardioembolic stroke: topography and pathogenesis. Cerebrovasc Brain Metab Rev. 1992;4(1):28-58.

22. Ho BL, Huang P, Khor GT, Lin RT. Simultaneous thrombosis of cerebral artery and venous sinus. Acta Neurol Taiwan. 2008;17(2):112-116.

23. Mehta PJ, Chapman S, Jayam-Trouth A, Kurukumbi M. Acute ischemic stroke secondary to iron deficiency anemia: a case report. Case Rep Neurol Med. 2012;2012:487080.

24. Azab SF, Abdelsalam SM, Saleh SH, Elbehedy RM, Lotfy SM, Esh AM, Srea MA, et al. Iron deficiency anemia as a risk factor for cerebrovascular events in early childhood: a case-control study. Ann Hematol. 2014;93(4):571-576.

25. Maguire JL, deVeber G, Parkin PC. Association between iron-deficiency anemia and stroke in young children. Pediatrics. 2007;120(5):1053-1057. 